\title{
HISTORIA DE LA PALEORNITOLOGÍA EN ESPAÑA A TRAVÉS DE LOS DOCUMENTOS CIENTÍFICOS
}

\author{
Antonio SÁNCHEZ MARCO' e Isabel SASTRE PÁEZ² \\ ' Departamento de Paleobiología. Museo Nacional de Ciencias Naturales. José Gutiérrez \\ Abascal, 2. 28006 Madrid. España. antoniosm@mncn.csic.es \\ 2 Hermanos Machado, 4. 28660 Boadilla. España.
}

Sánchez Marco, A. y Sastre Páez, I. 2001. Historia de la Paleornitología en España a través de los documentos científicos. [History of the Paleornithology in Spain through its scientific documents.] Revista Española de Paleontología, 16(1), 99-113. ISSN 0213-6937.

\begin{abstract}
The first document published in Spain on Spanish avian fossils was written by E. Harlé. These fossils were found in Bora Gran locality and their identification was realised by Milne- Edwards, naturalist and founder of the paleornithological studies. The finding of an eggshell from the Miocene outcrop of Cervico de la Torre was the subject of the first work due to a spanish student, M. de Olavarría, in 1898.

Several studies took place in the earliest years of the twentieth century. Among them, maybe, the most outstanding items were: E.T. Newton's identification -done about 1914- of the abundant fossil remains from El Castillo, published in 1984, the study of Quaternary collection from Devil's Tower by D. M. Bate in 1928, and L. Navás description of fossils from the Miocene locality of Libros in 1922. In the twenties and thirties, birds from some paleolitic sites in Basque country were identified by C. Gaillard.

J. F. de Villalta started a new stage in the decade of sixties, realizing a catalogue of the birds from the Miocene and another one from the Quaternary. In 1968, a study on a deposit from Gibraltar was published by A. Easham. Since then, she has worked on several Spanish localities. In the decade of eighties there was an increasing interest towards the birds from the past. The activity of J. Boessneck and A. von den Driesh on Holecene sites, and C. Mourer-Chauviré's on Pleistocene outcrops can be underlined. Although J. Boesssneck had already realised two studies in Spain in 1969 and 1973. During this period new researchers joined, enlarging remarkably the previous knowledge on this field. Some Cretaceous findings are studied -particularly relevant to the understanding of the early evolution of birds- as well as some outcrops of Balearic and Canary islands -connected with processes of insularity divergence- and Tertiary and Quaternary Iberian localities, putting paleozoogeographical, paleoclimatical, systematic and man-birds interactions problems.
\end{abstract}

Keywords: Paleornithology, science history, fossil birds, Quaternary, Tertiary, Cretaceous.

\section{RESUMEN}

El primer documento publicado donde se da cuenta de un hallazgo de restos fósiles de aves en España es obra de E. Harlé. La identificación de estos fósiles, hallados en la Bora Gran, se debió a A. Milne-Edwards, naturalista y fundador de los estudios paleornitológicos. Un artículo de M. de Olavarría fechado en 1898, acerca del hallazgo de un huevo en el yacimiento mioceno de Cevico de la Torre, es el primer trabajo de un español sobre restos de aves del pasado.

Después de ésto, quizá lo más relevante sea la identificación que hace E.T. Newton hacia 1914 de los abundantes restos fósiles del yacimiento cuaternario de El Castillo -publicado en 1984-, el estudio de D.M.A. Bate del depósito, así mismo de edad cuaternaria, de Devil's Tower en 1928 y las descripciones de L. Navás sobre fósiles de la localidad miocena de Libros en 1922. C. Gaillard, paleornitólogo del Museo de Historia Natural de Lyon estudió las aves de varios yacimientos paleolíticos vascos en los años veinte y treinta.

J.F. de Villalta comenzó en la década de los sesenta una nueva etapa. Confeccionó un catálogo de las aves del Mioceno y otro con las del Cuaternario. En 1968, A. Eastham publicó un estudio de un yacimiento de Gibraltar. A partir de entonces, ha trabajado en diversos yacimientos españoles. Es en la década de los años ochenta cuando empieza a producirse un incremento considerable en la atención a las aves del pasado. C. Mourer-Chauviré participa en dos proyectos de investigación españoles. También abarca toda esta década la actividad que encabezan J. Boessneck y A. von den Driesch en el marco del Instituto Arqueológico Alemán, quienes estudian 
yacimientos holocenos españoles, si bien el primero de los investigadores ya había realizado sendos estudios en 1969 y 1973. Este es también el periodo en que se incorporan nuevos investigadores, que amplían notablemente lo que se sabía en este campo. Se estudian yacimientos cretácicos -interesantes para el conocimiento de la evolución temprana de las aves-yacimientos de Baleares y Canarias -que se suman a los avances por comprender los fenómenos de insularidad- y localidades peninsulares terciarias y cuaternarias -que se insertan en problemáticas paleozoogeográfricas, paleoclimatológicas, sistemáticas, de las relaciones hombre-aves, etc.

\section{Palabras clave: Paleornitología, historia de la ciencia, aves fósiles, Cuaternario, Terciario, Cretácico.}

\section{INTRODUCCIÓN}

La biología y la geología han transformado la concepción que el hombre tiene de sí y de su mundo a partir del siglo XIX. Han provocado una de las revoluciones intelectuales más profundas que registra la historia, pues sus consecuencias no se limitan al ámbito de las ciencias naturales, sino también a la religión, a la filosofía, a la ética, etc. Dos son las nociones fundamentales que aportan al conocimiento general de la época industrial. Una es la de cambio; un cambio sin pausa, como uno de los rasgos esenciales del cosmos. La otra es la de tiempo, el de la gran antiguiedad de la Tierra y de la vida. Ambas, cambio y tiempo, dos modos de medir el movimiento.

Esta revolución intelectual, que se desarrolló con un ritmo muy rápido -y fue acompañada de cierta conmoción social- a partir de la segunda mitad del siglo XIX, estuvo precedida por un cambio o -visto retrospectivamente- una preparación terminológica. Ya en 1802 el término biology adquirió su significado actual de tratado de la vida en los trabajos de G.R. Treviranus (1802-1822) y, más tarde, en los de J.B. Lamarck. Pero el concepto de cambio o transmutación se comenzó a utilizar casi sincrónicamente en la embriología y en la paleontología. De hecho, fueron estas dos ramas de las ciencias naturales las que establecieron, siguiendo un camino tortuoso, en la atmósfera intelectual que fomentaba el idealismo alemán y, particularmente, en el terreno abonado por la Naturphilosophie, la tesis de la transmutación de las especies.

Esta aportación fundamental de la ciencia europea del siglo XIX al modo en que el hombre entiende la naturaleza era seguida por científicos españoles, pero no tuvieron una participación destacada. Las causas no fueron muy diferentes de las que se pueden deducir al observar la historia de una de las especialidades de la paleontología, la del estudio de las aves fósiles.

La paleornitología constituye un capítulo de la paleontología, el que se refiere a las aves. El objeto de estudio es el pasado de las aves; es decir, tanto las aves mismas que existieron en tiempos pretéritos, como los procesos de diversa índole en que se han visto implicadas (especiaciones, extinciones, adaptaciones, dispersiones, etc.). La arqueozoología estudia los restos de animales encontrados en yacimientos arqueológicos.

Este trabajo no es una lista comentada de los yacimientos paleornitológicos españoles. Todas las localidades del Terciario se pueden consultar en Sánchez (1995a y 1999a) y la mayoría de las del Cuaternario, en Villalta (1964), en Hernández (1993, 1994a) y en Tyrberg (1998).

Este artículo se atiene exclusivamente a los documentos publicados en revistas especializadas o en monografías y a tesis doctorales: no se mencionan libros de texto o de divulgación, ni artículos en diarios o semanarios. Tampoco se analizan las circunstancias sociales o académicas en que se dieron los primeros pasos en el estudio de las aves del pasado, si bien se mencionan algunas al final.

Establecer el punto en que comienza la historia de algo no es trivial. Un aspecto interesante en toda historia es la descripción y análisis del surgimiento del objeto de esa historia. La historia de algo empieza con su génesis, cuando aún no existe ese algo. Habida cuenta de que la paleornitología, tal como se entiende la ciencia moderna, se apoya convencionalmente en documentos editados por personas distintas del autor, y dado que este artículo es fundamentalmente bibliográfico, se ha asignado la inauguración de esta disciplina en España al primer trabajo original que trata sobre un hallazgo de fósiles avianos. La fecha es 1882 .

Desde sus comienzos hasta el presente, la paleornitología no ha atraído más que a un número muy reducido de practicantes, aunque el pasado de las aves muy pronto focalizó los debates en torno a los problemas de la evolución. 1861 fue el año en el que se descubrió la impresión de una pluma y el esqueleto casi completo del denominado "ejemplar de Londres" de Archaeopteryx lithographica Meyer, 1861. Dos años antes, había salido de imprenta "On the origin of Species by means of natural selection" (Darwin, 1859). El hallazgo de esta ave en sedimentos del Jurásico superior y su condición de "forma intermedia" entre los reptiles y las aves supuso una evidencia importante para los transformacionistas.

\section{LOS COMIENZOS}

En 1882 se publica una obra del prehistoriador francés E. Harlé sobre la fauna de un yacimiento magdaleniense de Gerona, la Bora Gran den Carreres. La identificación de los fósiles de aves fue realizada por A. MilneEdwards, naturalista que demostró en su tiempo una enorme capacidad de trabajo. Fue sin duda uno de los fundadores de los estudios paleornitológicos y también se 
cuenta entre los precursores de las ideas evolucionistas, alguna de cuyas obras fue escogida por C. Darwin como punto de apoyo con ocasión de la redacción de su obra más conocida y, después, durante los debates que se dieron a renglón seguido de su publicación. En esos años de los inicios de la paleornitología se están publicando también estudios precursores en la paleontología española. L. Mallada da a conocer entre 1875 y 1891 la "Sinopsis de las especies fósiles que se han encontrado en España" y que culmina en el "Catálogo general de las especies fósiles encontradas en España”, aparecido en 1892.

No obstante, el interés por el pasado de las aves estaba muy lejos de arraigar en España. Además, conviene hacer notar que hay algunos elementos diferenciadores entre los trabajos que se realizan con restos del Terciario y los que se hacen del Cuaternario.

\section{LOS COMIENZOS DEL ESTUDIO DEL TERCIARIO}

En cuanto al Terciario, la mayor parte de las obras de esta primera época sólo registran encuentros fortuitos de fósiles de aves, pero no hay interés por estudiar los especímenes que se han hallado casualmente. El número de publicaciones es muy reducido. En ellas, se menciona algún hallazgo y se da noticia de su ubicación geográfica y estratigráfica.

En 1898, M. de Olavarría da cuenta de un yacimiento con cáscaras de huevo en la localidad miocena de Cevico de la Torre (Palencia). Estos restos se recogen en un breve catálogo de vertebrados terrestres del Mioceno (Hernández-Pacheco, 1914a), junto con la mención de otro hueso procedente del yacimiento del Cerro del Otero, también en Palencia. Este autor da a conocer ese mismo año la aparición de cáscaras de huevo en el yacimiento también mioceno de La Puebla de Almuradiel (Toledo) (Hernández-Pacheco, 1914b). Un año después, se publica otro trabajo de E. Hernández-Pacheco y J. Dantin (1915) en el que se vuelve a tratar el Cerro del Otero.

Es poco lo que se hace en los años veinte. En un par de artículos, E. Hernández-Pacheco (1921 a, b) aparentemente revisa el material de La Puebla de Almuradiel y menciona que, además de las cáscaras de huevo ya conocidas, hay huesos de aves. Pero lo que puede ser valorado como el primer verdadero estudio de unas aves del Terciario ibérico se debe a L. Navás (1922 $\mathrm{a}, \mathrm{b}$ ). Este autor distingue una nueva especie en el famoso yacimiento mioceno de Libros. Varios años más tarde, F. Hernández-Pacheco (1929), hijo de E. HernándezPacheco, consagra un artículo a dos pistas de huellas de aves ("de pequeñas palmípedas y limícolas") de la localidad oligocena de Peralta de la Sal (Huesca). F. Hernández-Pacheco (1930), en un estudio regional se refiere al hallazgo de unos fósiles de aves, pero no ofrece indicación alguna del punto o puntos donde fueron encontrados. Sólo se sabe que su aparición tuvo lugar en la provincia de Valladolid, en sedimentos del Mioceno. Por otra parte, estos restos han desaparecido.

\section{LOS COMIENZOS DEL ESTUDIO DEL CUATERNARIO}

El interés que suscitan los fósiles de aves terciarias durante estas primeras décadas del siglo es, como se vé, escaso. No sucede exactamente igual en los estudios del Cuaternario, el periodo que se extiende desde hace 1,7 millones de años hasta el presente. Los prehistoriadores españoles y franceses que trabajan en yacimientos españoles comprenden que los datos paleoecológicos y paleoetnográficos que pueden suministrar los huesos de aves enriquecen la visión del entorno en que han transcurrido las etapas pretéritas de los seres humanos. Así, el conocimiento de las aves que reclaman las investigaciones prehistóricas -como de los otros animales existentes en los yacimientos con presencia humana- es esencialmente instrumental, por lo que no alcanza mucho más allá que el suficiente para inferir las condiciones exteriores en que se desarrolla la cultura humana.

E.T. Newton identifica a principios del siglo XX las aves de varios yacimientos cuaternarios: primeramente, Harlé (1908a) publica una corta lista con las especies de Hornos de la Peña (Santander), a la que se añaden dos artículos con las aves de dos localidades portuguesas, Das Fontainhas (Harlé, 1908b) y Furninha (Harlé, 1909, 1910). Mucho tiempo después, Cabrera (1984), en su monografía sobre El Castillo (Santander) publicó las determinaciones que había realizado Newton sobre los fósiles extraídos por $\mathrm{H}$. Obermaier en sus excavaciones de principios de la segunda década del siglo. Las etiquetas originales donde este autor consignaba los nombres de los taxones están fechadas por él mismo en 1914. El Castillo ha albergado el conjunto ornítico más rico de la península ibérica hasta que se ha conocido el de Atapuerca. En los años de la Gran Guerra europea, H. Breuil descubrió en el peñón de Gibraltar el abrigo de Devil's Tower, cuyas aves fueron encomendadas a E.T. Newton para su examen (Breuil, 1922). Transcurridos varios años, se publicó un nuevo estudio de la fauna de Devil's Tower, obra esta vez de D.M.A. Bate (1928), y no mucho más tarde, se inició una cierta colaboración de C. Gaillard con los prehistoriadores T. Aranzadi y J.M. Barandiarán. Anteriormente, Gaillard ya había ayudado al prehistoriador francés E. Passemard con los restos de aves procedentes de dos localidades paleolíticas del otro lado de la frontera, Isturitz y Olha (Passemard, 1924). Aranzadi y Barandiarán hacían selecciones de huesos de los yacimientos vascos que estaban estudiando y se las envíaban a C. Gaillard (Elorza, 1990). Primero apareció un artículo sobre el yacimiento de Ermittia (Aranzadi y Barandiarán, 1928), que fue seguido por otros trabajos dedicados al de Santimamiñe y al de Lumentxa (Aranzadi et al., 1931; Aranzadi y Barandiarán, 1935). P.R. Lowe polemiza con C. Gaillard acerca del pretendido hallazgo de restos de Phasianus que hace este autor en el yacimiento de Santimamiñe y en el de Pageyral (sur de Francia). El primero afirma que se trata de meros huesos de gallo doméstico (la discusión se puede consultar en Gaillard, 1926, y en Lowe, 1933). 
Ritmo lento en la aparición de los primeros trabajos y escasa participación de investigadores nativos, a lo que se añade que los objetivos no eran, por lo común, los de conocer las aves por sí mismas, marcaron los primeros pasos de la paleornitología en España.

\section{LA ETAPA INTERMEDIA}

Esta es una etapa en la que se desarrollan y plantean nuevas concepciones que ejercerán una gran influencia en todas las ciencias naturales. En la década de los cuarenta, un grupo de investigadores evolucionistas, entre los que destacaban T. Dobzhansky, E. Mayr, G.G. Simpson y J. Huxley, establecieron la "teoría sintética de la evolución". Los trabajos de varios de estos investigadores sobre la distribución geográfica de las aves y su registro fósil constituyen algunos de los argumentos más consistentes en la discusión que se desarrolla entonces. Esta labor se continuó hasta los años setenta, y de ella es heredera la práctica totalidad de los biólogos actuales. Aquellos autores rescataron las nociones centrales del darwinismo y las hicieron compatibles con el conjunto de conocimientos que se estaban adquiriendo en el campo de la genética, sobre la que se asentaba el cuerpo teórico dominante en biología y que amenazaba barrer buena parte de las ideas evolucionistas. Se debe a W. Hennig una innovación conceptual y metodológica que tendrá más repercusión entre los paleornitólogos que se dedican al Mesozoico que entre los que se ocupan del Cenozoico. Hennig (1950) presenta la sistemática filogenética o cladista en un libro que es considerado un hito en la sistemática biológica. No obstante, esta propuesta no adquiere auge en la comunidad científica hasta la siguiente década, con la aparición de otros artículos y la traducción inglesa del libro. En los años sesenta se anuncia la teoría biogeográfica insular, obra de R.H. MacArthur y E.O. Wilson (1967), un intento de explicar los patrones de diversidad faunística que se observan en las islas. Recibe críticas favorables (v.p.e. Vuilleumier, 1975), pero ha sido rechazada por paleornitólogos (v.p.e. Olson, 1978; Florit y Alcover, 1987b). El modelo que proponen MacArthur y Wilson se apoya en los conceptos de la dinámica de poblaciones actuales y en la selección natural. Cuenta con abundantes datos de faunas actuales; sin embargo, entre otros puntos débiles, el modelo es ahistórico, no reserva ningún lugar para las faunas insulares del pasado.

Lo que sabemos sobre la evolución de las aves sería muy distinto sin el marco que ofrece la deriva continental. A finales de los años sesenta, esta teoría, enunciada por A. Wegener en 1912 y rechazada formalmente por la mayoría de los especialistas de su tiempo, se consolidaba como la nueva ortodoxia. Poco después, van viendo la luz desarrollos teóricos, con diferentes tendencias, del paradigma de la teoría sintética. $\mathrm{Su}$ influencia es más difícil de valorar en la paleornitología -o, en general, en la paleontología-, pero es indudable su efecto en las concepciones de los investigadores.
Casi toda la actividad de los años cincuenta y sesenta recae en J.F. de Villalta, investigador que se involucra directamente en un amplio campo de intereses paleontológicos. Colaboró con Crusafont en la descripción de los restos del yacimiento cuaternario de l'Altissent (Villalta y Crusafont, 1950a), de varios huesos de galliformes de la localidad miocena de Hostalets de Pierola (Villalta y Crusafont, 1950b) y de una nueva especie hallada en El Fallol, también, como el de Hostalets, situado en la cuenca del Vallés-Penedés (Crusafont y Villalta, 1955). Así mismo, Villalta (1963) realizó una revisión de las localidades del Mioceno. A este trabajo incorporó nuevos especímenes fósiles y algunos de ellos fueron clasificados como nuevos taxones. E hizo algo similar para el Cuaternario: realizó un catálogo (Villalta, 1964) con lo que se había publicado previamente sobre las aves cuaternarias de yacimientos españoles, y en el que también incluyó algunos yacimientos nuevos estudiados por él (las cuevas: Negra, del Toll, de las Tuxuneras, del Reclau Viver, del Gegant y de Berroberria, así como el abrigo Romaní). Posteriormente, publicó una nota sobre el yacimiento del límite Plio-Pleistoceno de las islas Medas (Villalta, 1965).

Probablemente como consecuencia de su formación paleontológica, J.F. de Villalta mostró un interés genuino por las aves en sí mismas, más allá de su valor instrumental. Estudió e identificó las especies de algunos yacimientos del Cuaternario, pero no sólo por la ayuda que pudieran prestar para comprender las condiciones ambientales en que se desarrollaron las vidas de los grupos humanos cuyos restos aparecían en los mismos yacimientos.

En los años siguientes, hay menciones esporádicas de hallazgos de restos de aves en artículos que tratan sobre otros grupos animales. Se trata de encuentros casuales. En ocasiones, simplemente se indica que ha aparecido algún hueso aviano (e.g. Adrover, 1975, 1986), otras veces, se ofrece alguna identificación, en ocasiones tentativa (Koby y Spahni, 1956; Adrover et al.,1974; Altuna, 1977), pero nunca hay detrás ningún estudio del material hallado. Por estos años, Rothe (1964) describe dos tipos de cáscaras de huevo, encontradas en depósitos del Mioceno o Plioceno del norte de Lanzarote, como de avestruz y de aepyornítida, respectivamente. La existencia del segundo grupo de aves gigantes en las Canarias plantea un problema biogeográfico (véase Sauer y Rothe, 1972; García-Talavera, 1990). También en los años sesenta, Raaf et al. (1965) escriben una nota con la comunicación del hallazgo de icnitas en rocas del Oligoceno inferior de la zona de Liedena (Navarra).

\section{LA ETAPA ACTUAL DEL ESTUDIO DEL CUATERNARIO}

Si es difícil establecer cuándo comienza a dar sus primeros pasos la paleornitología española, y en este artículo hemos tomado el año 1882 de modo un tanto arbitrario, otro tanto se puede afirmar con respecto a los 
comienzos de la etapa actual. Incluso se podría discutir si existe un conjunto de características que individualice una etapa actual. No obstante, por razones de exposición es útil establecerla. Su inicio estaría marcado por los primeros estudios de investigadores que trabajan actualmente en esta especialidad. Una novedad de esta etapa es que se aborda el estudio de yacimientos insulares (islas Canarias y Baleares) y de las evidencias relacionadas con fenómenos de insularidad.

\section{LA ETAPA ACTUAL DE LOS ESTUDIOS SOBRE EL CUATERNARIO INSULAR}

P. Ballmann, ayudado por C. Smeenk, determina las aves de la cueva de Son Bauzà (Mallorca). El resultado se publica en un artículo firmado por Ballman y Adrover (1970). Unos años más tarde, C. Mourer-Chauviré, paleornitóloga del CNRS francés, comienza en 1975 a colaborar con investigadores españoles. En esta fecha se publica una corta lista de vertebrados del yacimiento mallorquín del Avenc de Na Corna (Mourer-Chauviré et al., 1975). En él se identifica la especie Grus antigone (véase también Northcote y Mourer-Chauviré, 1985, 1988). Posteriormente, estudia varios otros yacimientos de la misma isla (Mourer-Chauviré et al., 1977).

La misma autora, junto con otros investigadores, describe Tyto balearica, especie extinguida de lechuza, cuyos vestigios se habían localizado en algunos yacimientos de las Baleares; yacimientos fechados en el límite Plio-Pleistoceno (Mourer-Chauviré et al., 1980). Este trabajo es uno de los primeros de lo que más tarde será una línea de investigación sobre la evolución en condiciones insulares. Con el impulso de J.A. Alcover mantenido a lo largo de varios años, un grupo de investigadores estudiará varios yacimientos de los archipiélagos balear y canario, la mayor parte, de edad Cuaternaria. De este esfuerzo, resulta el descubrimiento de nuevas especies y de muchos rasgos de las paleoavifaunas de estas islas. Esto se recoge en las siguientes publicaciones: Alcover y Florit (1987), Florit y Alcover (1987a y b), Alcover (1989), Florit et al. (1989), McMinn et al. (1990, 1993), Alcover et al. (1992, 1994), Alcover y McMinn (1992, 1994), McMinn y Alcover (1992), Jaume et al. (1993), Encinas y Alcover (1997). Un sumario de las aves y yacimientos de las islas Gimnésicas (Mallorca y Menorca, principalmente) fue realizado por Seguí (1996). Este autor, así mismo, ha participado con investigadores ya mencionados en el análisis de las aves de cueva Moleta (Seguí et al., 1997) y en un estudio comparativo de las avifaunas insulares mediterráneas con las de Hawai (Seguí y Alcover, 1999). Además de los trabajos de este grupo y de los realizados previamente con cáscaras de huevo que habían sido encontradas en Lanzarote (Rothe, 1964; Sauer y Rothe, 1972), algunos investigadores canarios han emprendido una aproximación a los restos de aves fósiles por medio del inventario de sitios y taxones correspondientes de las islas Canarias (García-Talavera, 1990; Báez, 1992; Castillo et al., 1996). A partir de aquí, la atención se ha dirigido hacia la identificación y estudio de aves de algunos yacimientos (Rando, 1995a, 1995b; Rando y López, 1996; Rando et al., 1996 y 1997), incluyendo la identificación de una nueva especie (Rando et al., 1999). También se han dedicado a tratar aspectos de las interacciones entre las poblaciones humanas y las aves (Rando y Perera, 1994). Paralelamente, no deja de haber incursiones esporádicas por parte de otros estudiosos ante el hallazgo de especímenes de interés particular (Walker et al., 1990). Las especies marinas de algunos yacimientos, tanto insulares como continentales del Pleistoceno superior, han sido objeto de un artículo paleogeográfico de Tyrberg (1999) que contempla las costas del Mediterráneo y las del noreste del Atlántico.

\section{LA ETAPA ACTUAL DE LOS ESTUDIOS SOBRE EL CUATERNARIO CONTINENTAL}

En 1968 apareció publicado el estudio de Eastham sobre el yacimiento gibraltareño de Gorham's cave (Pleistoceno superior). Constituye su primera toma de contacto con las aves del Cuaternario de la península ibérica, contacto que ha mantenido hasta ahora.

Es también hacia estas fechas cuando comienza la labor en Iberia -fundamentalmente en España- de una serie de investigadores alemanes, agrupados en el Instituto Arqueológico Alemán, e interesados fundamentalmente en el Holoceno (edad del Bronce, colonización fenicia, etc.). Crean una revista específica, donde recogen todo lo que hacen aquí ("Studien über frühe Tierknochenfunde von der Iberischen Halbinsel"). De estos autores, son J. Boessneck y A.v.d. Driesch quienes mantienen la continuidad y a quienes se debe la mayor parte de los estudios. Las dos primeras de estas publicaciones datan del mismo año: una, sobre el Cerro del Real (Boessneck, 1969) y otra, sobre Cabezo Redondo (Driesch y Boessneck, 1969), las cuales son seguidas por las de Uerpmann (1971), Driesch (1972, 1973, 1982), Boessneck (1973), Uerpmann y Uerpmann (1973), Driesch y Boessneck (1976, 1980a, 1980b), Lauk (1976), Boessneck y Driesch (1980a, 1980b), Amberger (1985), Driesch et al. (1985), Milz (1986) y Friesch (1987).

C. Fuentes ha estudiado algunos yacimientos. Su primera publicación trataba de las aves de uno de los yacimentos del Pleistoceno español con más renombre, Ambrona (Aguirre y Fuentes, 1969). Fósiles de este yacimiento fueron también analizados por D. Jánossy - paleontólogo del Museo de Historia Natural de Budapest-, quien utilizó algunos de ellos para erigir una nueva especie de ganso (Anser subanser Jánossy, 1983). Este taxón fue discutido posteriormente por A. Sánchez (1990a). En su trabajo sobre los vertebrados de El Padul, Fuentes y Meijide (1970) encontraron una especie de ave. También se deben a estos autores el estudio de la cueva Horá (Fuentes y Meijide, 1975) y de la de El Pendo (Fuentes, 1980).

J. Estévez ha estudiado las aves de tres yacimientos del Pleistoceno superior: cueva S'Espasa (Estévez, 1976), 
Bora Gran -que, como se ha dicho anteriormente, fue el primer yacimiento estudiado en España- y Mollet I, las dos últimas en el marco de su tesis doctoral (Estévez, 1979).

C. Mourer-Chauviré, en colaboración con otros paleontólogos y arqueólogos españoles estudió las aves del sitio del Pleistoceno medio de Áridos 1 (MourerChauviré, 1980). Poco tiempo después, en 1983, se publicó en una revista francesa la tesis doctoral de $\mathrm{Ph}$. Vilette, que trataba sobre algunas localidades del Pleistoceno superior y del Holoceno, en su mayoría del sur de Francia y de Cataluña (Vilette, 1983). Los yacimientos españoles de este trabajo son: cova Fosca (Castellón) y los yacimientos catalanes de Bora Gran, Arbreda, Roc de la Melca y Cingle Vermell.

En otro plano, estas fueron las fechas en que se volvió a suscitar una intensa discusión entre modelos de extinciones catastróficas y graduales a partir de las evidencias de impacto de un bólido contra la Tierra en el límite K / T (Álvarez et al., 1980). El registro cretácico de las aves aún es demasiado escaso y disperso para analizar las consecuencias de este impacto sobre la evolución aviana.

Como se ha dicho anteriormente, prehistoriadores del País Vasco fueron precursores en incorporar la información que proporcionan las aves fósiles a la reconstrucción de los ambientes pretéritos donde se desarrollaron las primitivas poblaciones humanas. Es quizá esta tradición multidisciplinar, conjugada con la abundancia de yacimientos en cuevas, lo que ha determinado que en el norte de la península se haya realizado un número considerable de estudios de aves. En esta labor, la Sociedad de Ciencias Aranzadi (Aranzadi Zientzi Elkartea) dirigió su interés hacia las aves fósiles, en primer lugar, encomendando varios trabajos a A. Eastham y, posteriormente, a uno de sus miembros, M. Elorza. El primer trabajo que podemos atribuir en este campo a "la Aranzadi" es el de Altuna y Mariezkurrena (1983), acerca del hallazgo más antiguo de gallo doméstico en el País Vasco. También promovidos por esta asociación fueron los estudios de las cuevas de Ekain (Eastham, 1984), Erralla (Eastham, 1985), Amalda (Eastham, 1990), Urtao II (Elorza, 1989), de algunas localidades estudiadas por A. Eastham (Elorza, 1990), una revisión posterior de Ermittia (Elorza, 1993) y del asentamiento humano sobre la playa de Herriko Barra (Elorza y Sánchez, 1993).

Mencionamos a continuación los artículos que han aparecido recientemente sobre depósitos arqueológicos en cueva ubicados en la costa norte ibérica: La Riera (Eastham, 1986a), La Cuevona (Sánchez, 1986), Laminak II (Hernández, 1994b), Pico Ramos (Castaños y Hernández, 1995), Berroberria (Díez et al., 1995), Urratxa III (Elorza, 1997) y Pala da Vella (Fernández et al., 1996).

Cataluña cuenta con un considerable número de localidades con aves; algunas de ellas, aún pendientes de investigación, y bastantes otras, como se ha indicado antes, estudiadas a lo largo de la corta historia de la paleornitología española (Harlé, 1882; Villalta, 1964;
Estévez, 1979; Vilette, 1983). Los restos avianos del yacimiento holoceno del Cingle Vermell, primeramente estudiado por P. Vilette (1983), han sido objeto de un nuevo artículo (Vila et al., 1985). Recientemente, han aparecido varios trabajos sobre yacimientos catalanes escritos por L. Garcia: Cova 120 (Agustí et al., 1987), Arbreda (Garcia, 1995), varios sitios en Serinyà (Soler y Garcia, 1995; Garcia, 1997) y Culip VI (Garcia, 1998). La cueva G-1 de Grioteres ha sido estudiada por $\mathrm{M}$. Millán (1995).

En el resto de la región mediterránea, disponemos de información sobre poblaciones avianas cuaternarias. Los investigadores agrupados en el Instituto Arqueológico Alemán, mencionados anteriormente, desarrollaron parte de su actividad en las zonas oriental y sur de la Península. El registro fósil de la cueva de Nerja ha sido objeto de cinco trabajos (Boessneck y Driesch, 1980a; Eastham, 1986b; Hernández, 1995a y 1995b; Tyrberg y Hernández, 1995). También en la costa sur, se halla la cova Negra estudada por Eastham (1989)- y el yacimiento urbano Puerto 6 (Huelva) -estudiado por Aguilar y Hernández (1989)-. Muy recientemente, se ha dado a conocer la existencia de dos húmeros de rabilargo (Cyanopica cyanus) en dos yacimientos gibraltareños: las cuevas Gorham y Vanguard (Cooper, 2000). El hallazgo de esta especie ha sido criticado por Sánchez (2000a). En la cara este de la región mediterránea, A. Eastham estudió los restos encontrados en los yacimientos de Mallaetes, Parpalló y cueva del Volcán del Faro (Eastham in Davidson, 1989) y en El Salt (Eastham, 1988). En la región mediterránea, hay algunas otras localidades, además de las citadas anteriormente, aunque con carácter continental. Las aves de la cueva de Zafarraya (Pleistoceno superior) aparecen en dos artículos (Eastham, 1989; Hernández, 1994a). Otras cuatro localidades han sido estudiadas por A. Sánchez: cueva Ambrosio, (Sánchez, 1988), el abrigo del Tossal de la Roca (Cacho et al., 1995; 1998) y dos afloramientos del Pleistoceno medio e inferior, respectivamente, Huéscar 1 (Sánchez, 1989) y sierra de Quibas (Montoya et al., 1999; Sánchez, 1999c). En la región del Levante español, hay algunos otros sitios paleolíticos con interesantes conjuntos orníticos, como la cueva de Les Cendres (Badal et al., 1991; Villaverde et al., 1997). Parece que en las cuevas de Blaus y Beneito han sido encontrados y estudiados algunos restos de aves, pero estos estudios no se han publicado expresamente, sino que se hace referencia a ellos de modo parcial (Villaverde y Martínez, 1995). Dos yacimientos castellonenses más modernos que los anteriores, cova Puntassa y el poblado del puig de la Misericordia, han sido estudiados por Garcia (1996) y Castaños (1994), respectivamente.

Cabe mencionar los yacimientos de las regiones castellanas. El afloramiento mesopleistoceno de Pinilla ha suministrado una colección de fósiles de aves que fue estudiada por el paleornitólogo Z. Bocheński, del Instituto de Zoología Sistemática y Experimental de Cracovia. Tal estudio no ha sido publicado hasta ahora. Lo único que se conoce de esta localidad es una breve referencia (Alférez et al., 1982b). Es importante señalar 
que Hernández y Tyrberg (1999) encontraron algunos restos de grulla damisela en tres localidades, así como el hallazgo del cormorán pigmeo en un yacimiento medieval (Hernández et al., 1999). El resto de las localidades castellanas tienen edades más antiguas y sus aves han sido estudiadas por Sánchez: Torralba (Sánchez, 1990a) y Ambrona (Sánchez, 1990a; 1999b) son del Pleistoceno medio; Valdegoba (Díez et al., 1989), Jarama II y Jarama VI (Adán et al., 1995) son del Pleistoceno superior. Muy renombrado a causa de la abundancia de fósiles pertenecientes a dos especies de homínidos es el complejo de yacimientos situados en la sierra de Atapuerca, con fechas desde el Pleistoceno inferior al Neolítico. Su registro de aves está entre los más ricos, tanto en restos como en especies, del Pleistoceno europeo, y este grupo de localidades es, así mismo, el que cuenta con un mayor número de artículos sobre sus restos avianos, tanto dedicados exclusivamente a las aves (Sánchez, 1987a, 1987b, 1995b, 1999d, 1999e) o integrándolas con otros elementos del registro con un enfoque más amplio (Aguirre et al., 1987 y 1990; Bermúdez et al., 1995; Rosas et al., 1998 y 1999).

Los últimos años han sido los más intensos en el descubrimiento de yacimientos y en el estudio de sus colecciones óseas. Consecuentemente, han sido los más fértiles en producción literaria. Se han realizado no sólo textos referidos a yacimientos concretos, sino también con contenidos más generales. Entre estos, estarían los catálogos ya mencionados, una reconsideración de las aves de localidades musterienses (Eastham, 1989), consideraciones sobre el arte paleolítico a partir de la aparición de golondrinas como motivo gráfico (Eastham, 1988), dos trabajos; sobre rapaces en el Holoceno superior en España (Aguilár y Hernández, 1991 y 1993), otro sobre la introdućción del gallo doméstico también en España (Hernández, 1992), sobre las aves en asentamientos medievales (Hernández, 1991; Hernández y Aguilar, 1994), sobre las avifaunas holocenas (Hernández y Morales, 1995), sobre la climatología, ecología y zoogeografía a partir del registro pleistoceno de aves (Sánchez, 1996b) y el registro de las aves limícolas en España (McMinn et al., 1997). Hay un número considerable de yacimientos del Bronce y Hierro, incluso de épocas más recientes, que no han sido indicados. Éstos, junto con bastantes datos no publicados anteriormente por el autor, se pueden hallar en Hernández (1993, 1994a), quien ha efectuado la mayor parte de su investigación en el Laboratorio de Arqueozoología de la Universidad Autónoma de Madrid.

\section{LOS ESTUDIOS ACTUALES DE LAS AVES DEL TERCIARIO Y DEL CRETÁCICO}

El camino de la ciencia está pavimentado con interpretaciones erróneas. Si no fuera así, esta actividad no tendría ningún interés. Merece la pena mencionar el descubrimiento en los años sesenta, como un hecho curioso, de Cosesaurus aviceps Ellenberger y Villalta,
1974. Este fue el nombre que se dio a un esqueleto incompleto hallado en sedimentos del Triásico medio de Montral-Alcover (Tarragona). Durante un tiempo, fue tenido como un antecesor de los Neornithes (Ellenberger, 1977, 1978). Un estudio posterior ubicó esta especie en un clado reptiliano, en particular en el grupo de prolacértidos (Sanz y López-Martínez, 1984).

Las huellas de pisadas y las cáscaras de huevo se han conservado en muchos menos paquetes sedimentarios que los huesos. Las escasas icnitas que han aparecido no han sido estudiadas con mucho esmero. A las ya conocidas de Peralta de la Sal (Hernández-Pacheco, 1929) y de Liedena (Raaf et al., 1965), en esta etapa se sumaron las que Casanovas y Santafé (1982) atribuyeron al orden de los Ciconiiformes. Estas icnitas se hallaron en capas del Oligoceno de Agramunt. Más tarde, Antón et al. (1993) descubrieron nuevos rastros en sedimentos del Mioceno de Salinas de Añana. Respecto a las cáscaras de huevos, la única contribución en la actual etapa de la paleornitología española fue el estudio realizado por Mein y Dauphin (1995) sobre algunos restos encontrados en la localidad del Plioceno de La Gloria, que fueron identificados como tipo-Aepyornis, siendo Aepyornis un género extinguido de aves terrestres de gran talla, endémicas de Madagascar.

Desde el descubrimiento de $T$. balearica en las islas Baleares (Mourer-Chauviré et al., 1980), han ido apareciendo restos de esta especie fósil de lechuza en afloramientos continentales más antiguos -tanto en Francia como en España- (Mourer-Chauviré y Sánchez Marco, 1988). La especie actual Geronticus eremita se presenta en una de estas localidades, Almenara 1, que constituyó el tema de dos artículos de Sánchez (1996a y 1999c). Cheneval, paleornitólogo del CNRS de Lyon, encontró $T$. balearica en un interesante conjunto ornítico procedente del yacimiento del Mioceno de Aljezar B (Cheneval y Adrover, 1993). Olson, reputado especialista de la Smithsonian Institution, ha reestudiado la especie $T$. sociata Navás, 1922a, descrita en Libros (Olson, 1995). Recientemente se ha publicado un trabajo sobre la presencia de esta especie en la península Ibérica (Sánchez, 2000b) así como otro sobre los escasos restos de passeriformes conocidos hasta el presente (Sánchez et al., 2000). En el yacimiento de Córcoles se halló también un pequeño número de restos. Fueron estudiados por J. Cheneval, pero de ellos sólo sabemos la identificación de Miophasianus sp. (Alférez et al., 1982a).

No se había intentado recopilar la información sobre las aves del Terciario -con la excepción parcial del catálogo de las aves del Mioceno de Villalta (1963)-. En el marco de la confección de un censo de todos los yacimientos europeos del Terciario (Mlíkovský, 1995), se preparó un primer catálogo de todos los restos avianos y sus correspondientes localidades (Sánchez, 1995a). Posteriormente, Sánchez (1999a) ha elaborado una revisión actualizada, pero ya referida a la península Ibérica. Este mismo autor ha estudiado las rapaces nocturnas del Terciario español (Sánchez, 2001).

La colección de aves mejor conocida es la del Plioceno inferior de Layna, la cual ha sido objeto de 
varios estudios (Mourer-Chauviré y Sánchez Marco, 1988; Sánchez, 1990b; Sánchez et al., 2000).

Verdaderos restos avianos del Mesozoico comenzaron a aparecer en los años ochenta. Lacasa (1985) describió y clasificó algunas plumas de La Pedrera de Meià (Lérida), procedentes de capas datadas del Cretácico inferior. De las plumas de este yacimiento ya se hallan referencias en Ferrer (1954) y en Gómez Pallerola (1979). En este último artículo, el autor incluyó por error en la clase de las aves un esqueleto al que denominó Priscavolucris. Quizá la primera mención del descubrimiento de restos óseos de ave en esta localidad fue la que hizo Vidal (1902), al comentar la destrucción accidental de un esqueleto en el terreno. Un año después de su trabajo sobre las plumas, Lacasa (1986) publicó la noticia de un nuevo hallazgo en esta misma localidad de la sierra del Montsec. Esta vez, un esqueleto con plumas, denominado Noguerornis algunos años más tarde (Lacasa, 1989a). Estos interesantes especímenes fueron, así mismo, tema de dos artículos posteriores (Lacasa, 1989b y 1990).

Sólo hay otro afloramiento Mesozoico en España conocido hasta ahora; también con una edad del Cretácico inferior. Se trata de Las Hoyas (Cuenca). En 1988, se supo del hallazgo de un llamativo esqueleto, en buena parte articulado (Sanz et al., 1988), del tamaño de un gorrión (véase también Sanz, 1989). Sanz y Bonaparte (1992) a continuación describieron este espécimen y le dieron el nombre de Iberomesornis. El mismo año, se dio a conocer el descubrimiento de otro esqueleto en la misma localidad (Sanz y Buscalioni, 1992), que se denominó Concornis. Este taxón constituye el primer hallazgo en España de los Enantiornithes, grupo ampliamente difundido durante el Mesozoico (Sanz et al., 1995). Otros dos nuevos hallazgos posteriores permitieron arrojar luz sobre la evolución del vuelo (Sanz et al., 1996) y sobre el desarrollo ontogénico de las aves primitivas (Sanz et al., 1997).

\section{NOTAS FINALES}

Hoy, casi exactamente una centuria después de los comienzos de los estudios paleornitológicos en España, gozamos de la suficiente distancia para apreciar algunas características del desarrollo de esta especialidad científica.

Los comienzos de esta rama de la paleontología en nuestro país fueron muy tardíos si los comparamos con los de otros países europeos. Quizá había pocas probabilidades para que esta pequeña parcela de la paleontología se desarrollara de otro modo. Es posible que algunas de las causas haya que buscarlas en una sociedad empobrecida, con una hacienda pública también empobrecida y un estado complicado durante mucho tiempo en una larga sucesión de guerras coloniales, incapaz, tanto de mantener los últimos vestigios del antiguo imperio español, como de desembarazarse de ellos. La atención social y de las instituciones hacia la investigación básica era escasa -si es que hubo alguna-. A lo que se suma que desde siempre el estudio de la vida de las aves en el pasado sólo ha comprometido a un puñado de estudiosos. Sin embargo, en condiciones difíciles, los puñados se convierten en individuos. Así, durante la mayor parte de la primera mitad del siglo XX, el interés hacia esta rama de la ciencia lo alientan personas aisladas, con escasas conexiones con colegas extranjeros y sin discípulos que pudieran establecer una continuidad través del tiempo.

La vinculación que mantenían los investigadores con entidades científicas es un exponente del apoyo institucional a la investigación. No es objetivo de este trabajo abordar con detalle este aspecto, pero sí se puede avanzar algún comentario al respecto. La relación del factor humano sobre el institucional en cuanto al impulso sobre la paleornitología ha variado en España a lo largo de su corta historia. Durante los primeros tiempos, hasta el comienzo de la etapa actual, la mayor parte de quienes se ocuparon de estudiar las aves ocupaban puestos estables y remunerados en alguna institución científica, pero la última es distinta a este respecto. El soporte institucional ha pasado a ser parcial e inestable. En esta etapa, el incremento de investigadores ha imprimido un crecimiento tanto en el número de publicaciones paleornitológicas, como en la calidad de éstas, como en la amplitud de temas que abordan. Nunca antes, como ahora, el esfuerzo e interés personales han tenido tanto peso en la investigación que se realiza en España sobre las ornitofaunas del pasado y la evolución de las aves.

\section{AGRADECIMIENTOS}

Nuestro agradecimiento a M. Elorza, L. García, F. Hernández, A. Lacasa, J. Morales, J.L. Sanz, J. vand der Made y a un revisor anónimo, por sus comentarios y aportaciones al manuscrito.

\section{BIBLIOGRAFÍA}

Adán, G., Arribas, A., Barbadillo, J., Cervera, J., Estrada, R., García, M.A., Jordá, J.F., Pastor, J., Sánchez, B., Sánchez, A., Sanchiz, B. y Sesé, C. 1995. Prospecciones y excavaciones arquelógicas en el alto valle del Jarama (Valdesotos, Guadalajara, Castilla-La Mancha). In: Arquelogía en Guadalajara (Eds. R. Balbín, J. Valiente y M.T. Mussat). Serv. Publ. Junta Comunidades CastillaLa Mancha, Toledo, 111-124.

Adrover, R. 1975. Principales yacimientos paleomastológicos de la provincia de Teruel y su posición estratigráfica relativa. Trabajos sobre NeógenoCuaternario, 4, 31-48.

Adrover, R. 1986. Nuevas faunas de roedores en el MioPlioceno continental de la región de Teruel (España). Interés bioestratigráfico y paleoecológico. Instituto de Estudios Turolenses, Teruel, 423 pp.

Adrover, R., Aguirre, E., Heintz, E., Moissenet, E. y Morales, J. 1974. Teruel II. In: Coloquio Internacional sobre bioestratigrafía continental del Neógeno Superior y Cuaternario Inferior. Libro Guía (Eds. E. Aguirre y J. 
Morales). Consejo Superior de Investigaciones Científicas, Madrid, 69-83.

Aguilar, A. y Hernández, F. 1989. El ave del yacimiento tartésico de Puerto 6 (Huelva). Huelva Arqueológica, 1011, 271-273.

Aguilar, A. and Hernández, F. 1991. The genus Falco in the upper Quaternary of the Iberian Peninsula. In: Biology and conservation of small falcons (Eds. M.K. Nicholls and R. Clarke). The Hawk and Owl Trust, Londres, $217-$ 222.

Aguilar, A. y Hernández, F. 1993. La presencia de rapaces en yacimientos holocénicos de la Península Ibérica. Alytes, 6, 379-388.

Aguirre, E. y Fuentes, C. 1969. Los vertebrados fósiles de Torralba y Ambrona. Études sur le Quaternaire dans le monde. VIII Congr. INQUA, París, 433-437.

Aguirre, E., Arsuaga, J.L., Bermúdez de Castro, J.M., Carbonell, E., Ceballos, M., Díez, C., Enamorado, J., Fernández, Y., Gil, E., Martín, A., Martínez, I., Rosas, A., Sánchez, A. et Sánchez, B. 1987. Occupations humaines au Pléistocène moyen de la Sierra d'Atapuerca (Ibeas, Burgos, Espagne). L'Anthropologie, 91, 29-44.

Aguirre, E., Arsuaga, J.L., Bérmudez, J.M., Carbonell, E., Ceballos, M., Díez, C., Enamorado, J., Fernández, Y., Gil, E., Gracia, A., Martín, A., Martínez, I., Morales, J., Ortega, A.I., Rosas, A., Sánchez, A., Sánchez, B., Sesé, C., Soto, E. and Torres, T.J. 1990. The Atapuerca Sites and the Ibeas Hominids. Human Evolution, 5, 55-73.

Agustí, B., Alcalde, G., Burjachs, F., Buxó, R., Juan-Muns, N., Oller, J., Ros, M.T., Rueda, J.M. i Toledo, A. 1987. Dinàmica de la utilització de la Cova 120 per l'home en els darrers 6.000 anys. Sèrie monogràfica, 7. Centre d'Investigacions Arqueològiques de Girona, 159 pp.

Alcover, J.A. 1989. Les aus fòssils de la Cova de Ca $\mathrm{Na}$ Reia. Endins, 14/15, 95-100.

Alcover, J.A. y Florit, F. 1987. Una nueva especie de "Carduelis" (Fringillidae) de La Palma. Vieraea, 17, 7586.

Alcover, J.A. i Florit, X. 1989. Els ocells del jaciment arqueològic de la Aldea, Gran Canària. Bolletí del Institut Català d'Història Natural, 56, 47-55.

Alcover, J.A. i McMinn, M. 1992. Presència de l'àguila marina Haliaeetus albicilla (Linnaeus 1758) al jaciment espeleològic quaternari d'Es Pouàs (Sant Antoni de Portmany, Eivissa). Endins, 17/18, 81-87.

Alcover, J.A. and McMinn, M. 1994. Predators of vertebrates on islands. BioScience, 44, 12-18.

Alcover, J.A., Florit, F., Mourer-Chauviré, C. and Weesie, P.D.M. 1992. The avifaunas of the isolated Mediterranean islands during the middle and late Pleistocene. In: Papers in avian paleontology honoring Pierce Brodkorb (Ed. K.E. Campbell). Natural History Museum of Los Angeles County, sc. ser., 36, 237-283.

Alcover, J.A., McMinn, M. and Altaba, C.R. 1994. Eivissa: a pleistocene oceanic-like island in the Mediterranean. National Geographic Research and Exploration, 10, 236-238.

Alférez, F., Molero, G., Brea, P. y Santafé, J.V. 1982a. Precisiones sobre la geología, fauna, cronoestratigrafía y paleoecología del yacimiento mioceno de Córcoles. Real
Academia de Ciencias Exactas, Físicas y Naturales, 76 (2), 249-276.

Alférez, F., Molero, G., Maldonado, E., Bustos, V., Brea, P. y Buitrago, A.M. 1982b. Descubrimiento del primer yacimiento cuaternario (Riss-Würm) de vertebrados con restos humanos en la provincia de Madrid (Pinilla del Valle). Coloquios de la Cátedra de Paleontología, 37, 15-32.

Altuna, J. 1977. Apéndice: La fauna de la Cueva del Conde. Boletín de Estudios Asturianos, 90/91, 486-487.

Altuna, J. y Mariezkurrena, K. 1983. Los restos más antiguos de gallo doméstico en el País Vasco. Estudios de Arqueología Alavesa, 11, 381-386.

Álvarez, L.W., Álvarez, W., Asaro, F. and Michel, H.V. 1980. Extraterrestrial cause for the Cretaceous-Tertiary extinction. Science, 208, 1095-1108.

Amberger, G. 1985. Tierknochenfunde vom Cerro Macareno/Sevilla. Studien über frühe Tierknochenfunde von der Iberischen Halbinsel, 9, 76-105.

Antón, M., López, G. and Santamaría, R. 1993. Estudio preliminar de la icnofauna miocena del yacimiento de Salinas de Añana (provincia de Álava). Comunicaciones de las IX Jornadas de Paleontología, 23-28.

Aranzadi, T. y Barandiarán, J.M. 1928. Exploraciones prehistóricas en Guipúzcoa los años 1924 a 1927. Diput. Guipúzcoa, S. Sebastián, 48 pp.

Aranzadi, T. y Barandiarán, J.M. 1935. Exploraciones en la caverna de Santimamiñe (Basondo, Cortézubi). $3 a$. memoria.- Yacimientos azilienses y paleolíticos. Exploraciones en la caverna de Lumentxa (Lequeitio). Diput. Vizcaya, Bilbao, 135 pp.

Aranzadi, T., Barandiarán, J.M. y Eguren, E. 1931. Exploraciones en la caverna de Santimamiñe (BasondoCortézubi). 1a. y 2a. memorias. Diput. Vizcaya, Bilbao, $114 \mathrm{pp}$.

Badal, E., Bernabéu, J., Buxó, R., Dupré, M., Fumanal, M.P., Guillem, P., Martínez, R., Rodrigo, M.J. y Villaverde, V. 1991. Cuaternario litoral de la provincia de Alicante sector Pego-Moraira. Actas de la VIII reunión nacional sobre el Cuaternario, 21-78.

Báez, M. 1992. Zoogeography and evolution of the avifauna of the Canary islands. In: Papers in avian paleontology honoring Pierce Brodkorb (Ed. K.E. Campbell). Natural History Museum of Los Angeles County, sc. ser., 36 , 425-431.

Ballmann, P. y Adrover, R. 1970. Yacimiento paleontológico de la cueva de Son Bauzà (Mallorca). Acta Geológica Hispánica, 5 (2), 58-62.

Bate, D.M.A. 1928. The animal remains. L.H.D. Buxton, G.E. Smith y D.M.A. Bate. Excavations of a Mousterian rock-shelter at Devil's Tower, Gibraltar. Journal of the Royal Anthropological Institute of Great Britain and Ireland, 58, 92-111.

Bermúdez, J.M., Díez, J.C., Mosquera, M., Nicolás, E., Pérez, A., Rodríguez, J. y Sánchez, A. 1995. El nicho ecológico de los homínidos del Pleistoceno Medio de Atapuerca. Complutum, 6, 9-56.

Boessneck, J. 1969. Die Knochenfunde vom Cerro del Real bei Galera (Prov. Granada). Studien über frühe Tierknochenfunde von der Iberischen Halbinsel, 1, 1-42. 
Boessneck, J. 1973. Vogelknochen aus der phönizischen und römischen Niederlassung von Toscanos. Studien über frühe Tierknochenfunde von der Iberischen Halbinsel, 4, 101-105.

Boessneck, J. und Driesch, A. v.d. 1980a. Tierknochenfunde aus vier südspanischen Höhlen. Studien über frühe Tierknochenfunde von der Iberischen Halbinsel, 7, 1-83.

Boessneck, J. und Driesch, A. v.d. 1980b. Knochenfunde aus dem römischen Munigua (Mulva), Sierra Morena. Studien über frühe Tierknochenfunde von der Iberischen Halbinsel, 7, 160-185.

Breuil, H. 1922. Palaeolithic man at Gibraltar: new and old facts. Journal of the Royal Anthropological Institute of Great Britain and Ireland, 51, 46-54.

Cabrera, V. 1984. El yacimiento de la cueva de "El Castillo" (Puente Viesgo, Santander). Biblioteca Praehistorica Hispana, 22, 485 pp.

Cacho, C., Fumanal, M.P., López, P., López, J.A., Pérez, M., Martínez, R., Uzquiano, P., Arnanz, A., Sánchez, A., Sevilla, P., Morales, A., Roselló, E., Garralda, M.D. y García-Carrillo, M. 1995. El Tossal de la Roca (vall d'Alcalà, Alicante). Reconstrucción paleoambiental y cultura de la transición del Tardiglaciar al Holoceno inicial. Recerques del Museu d'Alcoi, 4, 11-101.

Cacho, C., Fumanal, M.P., López, P., López, J.A., Arnanz, A., Uzquiano, P., Pérez Ripoll, M., Sánchez, A., Morales, A. and Roselló, E. 1998. The transition from Magdalenian to Epipalaeolithic in the Spanish mediterranean: El Tossal de la Roca. Actas del XIII Congreso U.I.C.P.P. A.B.A.C.O. Edizioni, Forlì, Italia, 635-640.

Casanovas, M. L. y Santafé, J. V. 1982. Icnofauna oligocena de Agramunt (Lérida, España). Acta Geologica Hispanica, 1-2 (17), 113-119.

Castaños, P. 1994. Estudio de los restos óseos. In: El poblado ibérico del Puig de la Misericordia de Vinaròs (Ed. A. Oliver). Associació Cultural Amics de Vinaròs, 155-184.

Castaños, P. y Hernández, F. 1995. Estudio de la fauna de aves de la cueva de Pico Ramos (Muskiz, Bizkaia). Munibe (Antropologia - Arkeologia), 47, 183-186.

Castillo, C., López, M., Martín, M. y Rando, J.C. 1996. La paleontología de vertebrados en Canarias. Revista Española de Paleontología, num. extr., 237-247.

Cheneval, J. et Adrover, R. 1993. L'avifaune du Miocène supérieur d'Aljezar B (Los Aljezares, province de Teruel, Espagne). Systématique et paléoécologie. Paleontologia i Evolució, 26-27, 133-144.

Cooper, J.H. 2000. First fossil record of Azure-winged Magpie Cyanopica cyanus in Europe. Ibis, 142, 150-151.

Crusafont, M. y Villalta, J. F. 1955. Parte Paleontológica. Apéndice I. Aves. In: El Burdigaliense continental de la cuenca del Vallés-Penedés (Eds. M. Crusafont, J. F. de Villalta y J. Truyols). Memorias y Comunicaciones del Instituto Geológico, 12, 236-237.

Darwin, C. 1859. On the origin of species by means of natural selection. Murray, Londres, $502 \mathrm{pp}$.

Davidson, I. 1989. La economía de final del Paleolítico en la España oriental. Diput. Prov. Valencia, Trabajos varios del S.I.P., 85 pp.
Díez, C., García, M.A., Gil, E., Jordá, J.F., Ortega, A.I., Sánchez, A. y Sánchez, B. 1989. La cueva de Valdegoba (Burgos). Primera campaña de excavaciones. Zephyrus, 51-52, 3-22.

Díez, C., Sánchez, A. y Moreno, V. 1995. Grupos avicaptores del Tardiglaciar: las aves de Berroberria. Munibe (Antropologia - Arkeologia), 47, 55-74.

Driesch, A.v.d. 1972. Östearchäologische Untersuchungen auf der Iberischen Halbinsel. Studien über frühe Tierknochenfunde von der Iberischen Halbinsel, 3, 267 pp.

Driesch, A.v.d. 1973. Nahrungsreste tierischer Herkunft aus einer tartessischen und einer spätbronzezeitlichen bis iberischen Siedlung in Südspanien. Studien über frühe Tierknochenfunde von der Iberischen Halbinsel, 4, 9-31.

Driesch, A.v.d. 1982. Vogelknochen aus dem kupferzeitlichen Valencina de la Concepción/Sevilla. Studien über frühe Tierknochenfunde von der Iberischen Halbinsel, 8 , 179-184.

Driesch, A.v.d. und Boessneck, J. 1969. Die Fauna des "Cabezo Redondo" bei Villena (Prov. Alicante). Studien über frühe Tierknochenfunde von der Iberischen Halbinsel, 1, 43-89.

Driesch, A.v.d. und Boessneck, J. 1976. Die Fauna vom Castro do Zambujal. Studien über frühe Tierknochenfunde von der Iberischen Halbinsel, 5, 4-129.

Driesch, A.v.d. und Boessneck, J. 1980a. Die Motillas von Azuer und Los Palacios (prov. Ciudad Real). Untersuchung der Tierknochenfunde. Studien über frühe Tierknochenfunde von der Iberischen Halbinsel, 7, 84121.

Driesch, A.v.d. und Boessneck, J. 1980b. Tierknochenfunde aus Sacaojos bei La Bañeza (Prov. León). Studien über frühe Tierknochenfunde von der Iberischen Halbinsel, 7, 122-159.

Driesch, A.v.d., Boessneck, J., Kokabi, M. und Schäffer, J. 1985. Tierknochenfunde aus der Bronzezeitlichen Höhensiedlung Fuente Alamo, Provinz Almería. Studien über frühe Tierknochenfunde von der Iberischen Halbinsel, 9, 1-75.

Eastham, A. 1968. The avifauna of Gorham's Cave, Gibraltar. Bulletin of the London Institute of Archaeology, 7, 37-42.

Eastham, A. 1984. The avifauna of the cave of Ekain. In: El yacimiento prehistórico de la cueva de Ekain (Deba, Guipúzcoa) (Eds. J. Altuna y J.M. Merino). Eusko Ikaskuntza, 331-344.

Eastham, A. 1985. The Magdalenian avifauna at Erralla cave. Munibe (Antropología-Arqueología), 37, 59-80.

Eastham, A. 1986a. The La Riera avifauna. Anthropological Research Papers, 36, 275-284.

Eastham, A. 1986b. The birds of the Cueva de Nerja. In: La prehistoria de la Cueva de Nerja (Málaga) (Ed. J.F. Jordá). Patronato de la Cueva de Nerja, 109-131.

Eastham, A. 1988. The season or the symbol: the evidence of swallows in the Palaeolithic of Western Europe. Archaeozoologia, 2, 243-252.

Eastham, A. 1989. Cova Negra and Gorham's Cave: evidence of the place of birds in Mousterian communities. In: The walking larder: patterns of 
domestication, pastoralism and predation (Ed. J. Clutton-Brock). Unwin Hyman, London, 350-357.

Eastham, A. 1990. The bird bones in the cave of Amalda. In: La Cueva de Amalda (Zestoa, País Vasco): ocupaciones paleolíticas y postpaleolíticas (Eds. J. Altuna, A. Baldeón y K. Mariezkurrena). Eusko Ikaskuntza, 239253.

Ellenberger, P. 1977. Quelques précisions sur l'anatomie et la place systématique très spéciale de Cosesaurus aviceps (Ladinien supérieur de Montral, Catalogne). Cuadernos de Geología Ibérica, 4, 169-188.

Ellenberger, P. 1978. L'origine des oiseaux. Historique et méthodes nouvelles. Les problèmes des Archaeornithes. La venue au jour de Cosesaurus aviceps (Mushelkalk supérieur). Mémoires et Travaux E.P.H.E. de l'Institut de Montpellier, 4, 91-117.

Ellenberger, P et Villalta, J.F. 1974. Sur la présence d'un ancêtre probable des oiseaux dans le Muschelkalk supérieur de Catalogne (Espagne). Note préliminaire. Acta Geologica Hispanica, 9, 162-168.

Elorza, M. 1989. Avifauna de la cueva sepulcral de Urtao II (Oñati, Guipúzcoa). Munibe (Antropologia-Arkeologia), 41, 79-80.

Elorza, M. 1990. Restos de aves en los yacimientos prehistóricos vascos. Estudios realizados. Munibe (Antropologia-Arkeologia), 42, 263-267.

Elorza, M. 1993. Revisión de la avifauna de Ermittia (Guipuzkoa). Munibe (Antropologia-Arkeologia), 45, 175-177.

Elorza, M. 1997. La avifauna del yacimiento de Urratxa III (Orozko, Bizkaia). In: El yacimiento de la cueva de Urratxa III (Orozko, Bizkaia) (Eds. M. Muñoz y E. Berganza). Universidad de Deusto, Bilbao, 191-205.

Elorza, M. and Sánchez, A. 1993. Postglacial fossil Great Auk and associated avian fauna from the Biscay Bay. Munibe (Antropologia-Arkeologia), 45, 179-185.

Encinas, J.A. i Alcover, J.A. 1997. El jaciment fossilifer de la cova Estreta (Pollença). Endins, 21, 83-92.

Estévez, J. 1976. Hallazgo de una pantera en el Pleistoceno catalán. Speleon, 22, 171-178.

Estévez, J. 1979. La fauna del Pleistoceno catalán. Tesis doctoral (inédita). Universidad de Barcelona, 517 pp.

Fernández, C., Villar, R., Varela, P., Rey, J.M. y Elorza, M. 1996. Primeros datos cronológicos y paleontológicos del yacimiento de Pala da Vella (Biobra, Ourense). In: Biogeografía Pleistocena-Holocena de la península ibérica (Coords. P. Ramil, C. Fernández y M. Rodríguez). Conselleria de Cultura, Santiago, 249-261.

Ferrer, L. 1954. Notice préliminaire concernant la présence d'une plume d'oiseau dans le Jurassique supérieur du Montsech (Province de Lerida, Espagne). Acta XI Congressus Internationalis Ornithologici, 268-269.

Florit, X. i Alcover, J.A. 1987a. Els ocells del Pleistocè superior de la cova Nova (Capdepera, Mallorca). I. El registre. Bolletí de la Societat d'Història Natural dels Balears, 31, 7-32.

Florit, X. i Alcover, J.A. 1987b. Els ocells del Pleistocè superior de la cova Nova (Capdepera, Mallorca). II. Fauna associada i discussió. Bolletí de la Societat d'Història Natural dels Balears, 31, 33-44.
Florit, X., Mourer-Chauviré, C. i Alcover, J.A. 1989. Els ocells pleistocènics d'Es Pouàs, Eivissa. Nota preliminar. Bolletí del Institut Català d'Història Natural, 56, 35-46.

Friesch, K. 1987. Die Tierknochenfunde von Cerro de la Encina bei Monachil, Provinz Granada (Grabungen 1977-1984). Studien über frühe Tierknochenfunde von der Iberischen Halbinsel, 11, 135 pp.

Fuentes, C. 1980. Estudio de la fauna de El Pendo. Bibliotheca Praehistorica Hispana, 17, 217-237.

Fuentes, C. y Meijide, M. 1970. Estudio de los vertebrados fósiles de la turbera de El Padul (Granada). Boletín de la Real Sociedad Española de Historia Natural (Biología), 68, 199-206.

Fuentes, C. y Meijide, M. 1975. Fauna fósil de la cueva Horá (Granada). Estudios geológicos, 31, 777-784.

Gaillard, C. 1926. L'origine du faisan d'Europe. Bolletí de l'Associació Catalana d'Antropologia, Etnologia $i$ Prehistoria, 1926, 80-84.

Garcia, L. 1995. Preliminary study of Upper Pleistocene bird from bone remains from l'Arbreda cave (Catalonia). Courier Forschungsinstitut Senckenberg, 181, 215-227.

Garcia, L. 1996. Los restos de aves. In: Campaña de excavaciones en el yacimiento del calcolítico-bronce de Cova Puntassa (Castellón) (Eds. V. Palomar y F. Gusi). Quaderns de Prehistòria i Arqueologia de Castelló, 17, 124-125.

Garcia, L. 1997. Les restes d'oiseaux des sites de Serinyà (Pays Catalans). In: El món mediterrani després del Pleniglacial (18.000-12.000 BP) (Eds. J.M. Fullola i N. Soler). Museu d'Arqueologia de Catalunya, Girona, 329-344.

Garcia, L. 1998. Les restes d'au del jaciment suaquàtic de Culip VI. In: Excavacions arqueològicques subaquàtiques a Cala Culip 2, Culip VI (Eds. X. Nieto y X. Raurich). Girona, 259-260.

García-Talavera, F. 1990. Aves gigantes en el Mioceno de Famara (Lanzarote). Revista Academia Canaria de las Ciencias, 2, 71-79.

Gómez Pallerola, J.E. 1979. Un ave y otras especies fósiles nuevas de la biofacies de Santa María de Meyá (Lérida). Boletín Geológico y Minero, 90 (4), 333-346.

Harlé, E. 1882. La grotte de Serinyà, près de Gérone (Espagne). Matériaux pour l'Histoire de l'Homme, 13, 293-2992.

Harlé, E. 1908a. Faune quaternaire de la province de Santander (Espagne). Bulletin de la Société géologique de France, sér. 4, 8, 300-302.

Harlé, E. 1908b. Faune de la grotte Das Fontainhas (Portugal). Bulletin de la Société géologique de France, sér. 4, 8, 460-466.

Harlé, E. 1909. Faune de la grotte à Hyènes Rayées de Furninha et d'autres grottes de Portugal. Bulletin de la Société géologique de France, sér. 4, 9, 85-99.

Harlé, E. 1910. Les mammifères et oiseaux quaternaires connus jusqu'ici en Portugal. Mémoire suivi d'une liste générale de ceux de la Péninsule Ibérique. Comunicaçoes do Serviço Géologico de Portugal, 8, 22-86.

Hennig, W. 1950. Grundzüger einer Theorie der phylogenetischen Systematik. Deutcher Zentralverlag, Berlín. (Trad. 1979. Phylogenetic systematics. University of Illinois Press, 263 pp.) 
Hernández, F. 1991. Las aves del yacimiento de Angosta de los Mancebos (Madrid). Boletín de Arqueología Medieval, 5, 181-191.

Hernández, F. 1992. Some comments on the introduction of domestic fowl in Iberia. Archaeofauna, 1, 45-53.

Hernández, F. 1993. Catálogo provisional de los yacimientos con aves del Cuaternario de la península Ibérica. Archaeofauna, 2, 231-275.

Hernández, F. 1994a. Addenda al catálogo de yacimientos con aves del Cuaternario de la península Ibérica. Archaeofauna, 3, 77-92.

Hernández, F. 1994b. La avifauna no passeriforme de Laminak II. Kobie, ser. Paleoantropología, 21, 189-202.

Hernández, F. 1995a. Cueva de Nerja (Málaga): las aves de las campañas de 1980 y 1982. Trabajos sobre la cueva de Nerja, 5, 221-293.

Hernández, F. 1995b. El papel de las aves en la reconstrucción paleoecológica. Su aplicación al Cuaternario del sur de la península ibérica. Tesis doctoral (inédita). Universidad Autónoma de Madrid. $250 \mathrm{pp}$.

Hernández, F. y Aguilar, A. 1994. ¿Cristiano o musulmán? Las aves en los asentamientos medievales. Actas dos trabalhos de Antropologia e Etnologia, 34 (1/2), 443446.

Hernández, F. and Morales, A. 1995. Mid- and Late Iberian Holocene avifaunas: a global perspective. Courier Forschunginstitut Senckenberg, 181, 229-239.

Hernández, F. and Tyrberg, T. 1999. The demoiselle crane Anthropoides virgo in the Iberian peninsula, a summary of historical and subfossil data. Ardeola, 46, 97-100.

Hernández, F., Tyrberg, T. and von den Driesch, A. 1999. A record of pygmy cormorant Microcarbo pygmaeus from medieval Spain. Ardea, 87 (2), 285-288.

Hernández-Pacheco, E. 1914a. Los vertebrados terrestres del Mioceno de la Península Ibérica. Memorias de la Real Sociedad Española de Historia Natural, mem. 4, 9, 443488.

Hernández-Pacheco, E. 1914b. Mioceno superior de La Puebla de Almuradiel (Toledo). Boletín de la Real Sociedad Española de Historia Natural, 14, 274-278.

Hernández-Pacheco, E. 1921a. La llanura manchega y sus mamíferos fósiles (yacimiento de La Puebla de Almoradier). Comisión de Investigaciones Paleontológicas y Prehistóricas, mem. 28, 43 pp.

Hernández-Pacheco, E. 1921b. Nuevos yacimientos de vertebrados miocenos y deducciones de orden paleofisiográfico. Asociación Española para el Progreso de las Ciencias, 6, 159-170.

Hernández-Pacheco, F. 1929. Pistas de aves fósiles en el Oligoceno de Peralta de la Sal (Lérida). Memorias de la Real Sociedad Española de Historia Natural, 15, 379-382.

Hernández-Pacheco, F. 1930. Fisiografía, Geología y Paleontología del territorio de Valladolid. Comisión de Investigaciones Paleontológicas y Prehistóricas, mem. 37, 205 pp.

Hernández-Pacheco, E. y Dantin, J. 1915. Geología y paleontología del Mioceno de Palencia. Comisión de Investigaciones Paleontológicas y Prehistóricas, 5, 295 pp.
Jánossy, D. 1983. Die mittelpleistozäne Vogelfauna von Prezletice bei Prag (CSSR). Schriftenreihe der geologischen Wissenschaften, 19/20, 247-269.

Jaume, D., McMinn, M. and Alcover, J.A. 1993. Fossil birds from the Bujero del Silo, La Gomera (Canary islands), with a description of a new species of quail (Galliformes: Phasianidae). Boletin do Museu Municipal do Funchal, sup 2, 147-165.

Koby, F.E. et Spahni, C. 1956. Découverte dans le quaternaire espagnol d'un petit hamster: Allocricetus bursae Schaub. Eclogae Geologicae Helvetiae, 49, 543545.

Lacasa, A. 1985. Nota sobre las plumas fósiles del yacimiento eocretácico de La Pedrera-La Cabruna en la sierra del Montsec (prov. Lleida, España). Ilerda, 46, 227-238.

Lacasa, A. 1986. Nota preliminar sobre el hallazgo de restos óseos de un ave fósil en el yacimiento neocomiense del Montsec. Prov. de Lérida. España. Ilerda, 47, 203-206.

Lacasa, A. 1989a. Nuevo género de ave fósil del yacimiento neocomiense del Montsec (provincia de Lérida, España). Estudios geológicos, 45, 417-425.

Lacasa, A. 1989b. An early Cretaceous fossil bird from Montsec mountain (Lleida, Spain). Terra Nova, 1, 45-46.

Lacasa, A. 1990. Fósiles de las calizas litográficas del Montsec. Investigación y Ciencia, Marzo, 34-38.

Lauk, H.D. 1976. Tierknochenfunde aus bronzezeitlichen Siedlung bei Monachil und Purullena (Provinz Granada). Studien über frühe Tierknochenfunde von der Iberischen Halbinsel, 6, 117 pp.

Lowe, P.R. 1933. Differential characters in the tarsometatarsus of Gallus and Phasianus. Ibis, 1933 , 332-342.

MacArthur, R.H., and Wilson, E.O. 1967. The theory of island biogeography. Princeton University Press, Princeton, 203 pp.

Mallada, L. 1875 a 1891. Sinopsis de las especies fósiles que se han encontrado en España. Boletín del Mapa Geológico, tomos 2 a 17.

Mallada, L. 1892. Catálogo general de las especies fósiles encontradas en España. Boletín de la Comisión del Mapa Geológico de España, 1892, 1-253.

McMinn, M. i Alcover, J.A. 1992. Els ocells del Pleistocè superior de la Cova Nova (Capdepera, Mallorca). III. Noves aportacions al registre. Bolletí de la Societat d'Història Natural de les Balears, 35, 17-31.

McMinn, M. , Jaume, D. i Alcover, J.A. 1990. Puffinus olsoni n. sp.: nova espècie de baldritja recentment extinguida provinent de depòsits espeleològics de Fuerteventura i Lanzarote (Illes Canàries, Atlàntic Oriental). Endins, 16, 63-71.

McMinn, M., Altaba, R. i Alcover, J.A. 1993. La fauna fòssil de la cova den Jaume Orat (parròquia d'Albarca, Sant Antoni de Portmany, Eivissa). Endins, 19, 49-54.

McMinn, M., Sánchez Marco, A. y Alcover, J.M. 1997. Limícolas fósiles de la península ibérica e islas baleares. In: Las aves limícolas en España (Coord. A. Barbosa). Colección Técnica, Ministerio de Medio Ambiente, Madrid, 23-33.

Mein, P. et Dauphin, Y. 1995. Des coquilles d'oeufs de type 
Aepyornis dans le Bassin de Teruel (Pliocène basal, Espagne). Neues Jahrbuch für Geologie und Paläontologie Monatshafte, 3, 182-191.

Meyer, H.von 1861. Vögel-Federn und Palpipes priscus von Solnhofen. Neues Jahrbuch für Mineralogie, Geologie und Paläontologie, 1861, 561.

Millán, M. 1995. Estudi de la fauna de la cova G-1 de Grioteres. In: Les coves prehistóriques de les Grioteres (Vilanova de Sau-Osona-) (Ed. J. Castany). Sèrie Monografies, 16. Patronat d'Estudis Osonencs, Vic, 130151.

Milz, H. 1986. Die Tierknochenfunde aus drei argarzeitlichen Siedlung in der Provinz Granada (Spanien). Studien über frühe Tierknochenfunde von der Iberischen Halbinsel, 10, 149 pp.

Mlíkovský, J. 1995. Tertiary avian localities of Europe. Acta Universitatis Carolinae Geologica, 39, 519-528.

Montoya, P., Alberdi, M.T., Blázquez, A.M., Barbadillo, L.J., Fumanal, M.P., Made, J.van der, Marín, J.M., Molina, A., Morales, J., Murelaga, X., Peñalver, E., Robles, F., Ruiz, A., Sánchez, A., Sanchiz, B., Soria, D. y Szyndlar, Z. 1999. La fauna del Pleistoceno inferior de la sierra de Quibas (Abanilla, Murcia). Estudios Geológicos, 55, 127-161.

Mourer-Chauviré, C. 1980. Las aves del sitio de ocupación achelense de Áridos-1 (Arganda, Madrid). In: Ocupaciones achelenses en el Valle del Jarama (Eds. M. Santonja, N. López y A. Pérez). Publ. Excma. Diput. Prov. Madrid, Madrid, 145-160.

Mourer-Chauviré, C. et Sánchez Marco, A. 1988. Présence de Tyto balearica (Aves, Strigiformes) dans des gisements continentaux du Pliocène de France et d'Espagne. Geobios, 21, 639-644.

Mourer-Chauviré, C., Adrover, R. et Pons, J. 1975. Présence de Grus antigone (L.) dans l'Avenc de Na Corna a Majorque (Espagne). Nouveaux Archives du Muséum d'Histoire naturelle de Lyon, 13, suppl., 45-50.

Mourer-Chauviré, C., Moyà, S. et Adrover, R. 1977. Les oiseaux des gisements quaternaires de Majorque. Nouveaux Archives du Muséum d'Histoire naturelle de Lyon, 15, suppl., 61-64.

Mourer-Chauviré, C., Alcover, J.A., Moyà, S. et Pons, J. 1980. Une nouvelle forme insulaire d'effraie géante, Tyto balearica n. sp. (Aves, Strigiformes), du Plio-Pléistocène des Baléares. Geobios, 13, 803-811.

Navás, L. 1922a. Algunos fósiles de Libros (Teruel). Boletín de la Sociedad Ibérica de Ciencias Naturales, 21, 52-61.

Navás, L. 1922b. Algunos fósiles de Libros (Teruel): adiciones y correcciones. Boletín de la Sociedad Ibérica de Ciencias Naturales, 22, 172-174.

Northcote, E.M. and Mourer-Chauviré, C. 1985. The distinction between the extinct pleistocene European crane, Grus primigenia and the extant Asian Sarus crane, G. antigone. Geobios, 18, 877-881.

Northcote, E.M. and Mourer-Chauviré, C. 1988. The extinct crane Grus primigenia Milne-Edwards in Majorca (Spain). Geobios, 21, 201-208.

Olavarría, M. 1898. Huevos fósiles encontrados en Cevico de la Torre (Provincia de Palencia). Boletín de la Comisión del Mapa Geológico de España, 23, 133-138.
Olson, S.L. 1978. A paleontological perspective of West Indian birds and mammals. Zoogeography in the Caribbean, spec. publ. 13, 99-117.

Olson, S.L. 1995. Thiornis sociata Navás, a nearly complete miocene Grebe (Aves: Podicipedidae). Courier Forschungsinstitut Senckenberg, 181, 131-140.

Passemard, E. 1924. Les stations paléolithiques du Pays Basque et leurs relations avec les terraces d'alluvions de la Nive. Bodiou, Bayone, 218 pp.

Raaf, J.F.M. de, Beets, C. and Sluijs, G.K.van der 1965. Lower oligocene bird-tracks from northern Spain. Nature, 207, 146-148.

Rando, J.C. 1995a. Restos de hubara, Chlamydotis undulata (Jacquin, 1784) (Aves: Otididae), en la cueva del Viento (Tenerife, Islas Canarias). Vieraea, 24, 190-191.

Rando, J.C. 1995b. Presencia de restos de pigargo (Haliaeetus sp.) (Aves: Accipitridae) en yacimientos paleontológicos de Fuerteventura. Vieraea, 24, 65-69.

Rando, J.C. y López, M. 1996. Un nuevo yacimiento de vertebrados fósiles en Tenerife (Islas Canarias). $7^{\text {th }}$ International Symposium on Vulcanospeleology, 171173.

Rando, J.C. y Perera, M.A. 1994. Primeros datos de ornitofagia entre los aborígenes de Fuerteventura (islas Canarias). Archaeofauna, 3, 13-19.

Rando, J.C., Rodríguez, A.C., País, F.J., Navarro, J.F. y Martín, E. 1996. Los restos de aves del yacimiento arqueológico de "El Tendal" (La Palma, Islas Canarias). El Museo Canario, 51, 87-102.

Rando, J.C., López, M. and Jiménez, M.C. 1997. Bird remains from the archaeological site of Guinea (El Hierro, Canary Islands). International Journal of Osteoarchaeology, 7, 298-302.

Rando, J.C., López, M. and Seguí, B. 1999. A new species of extinct flightless passerine (Emberizidae: Emberiza) from the Canary Islands. The Condor, 101, 1-13.

Rosas, A., Carbonell, E., Cuenca, G., García, N., Fernández, Y., Made, J.van der, Ollé, A., Parés, J.M., Pérez, A., Sánchez, A., Sánchez, B. y Vallverdú, J. 1998. Cronología, bioestratigrafía y paleoecología del Pleistoceno Medio de Galería (sierra de Atapuerca, España). Revista Española de Paleontología, 13, 71-80.

Rosas, A., Carbonell, E., Ollé, A., Pérez, A., Vallverdú, J., Huguet, R., Cáceres, I., Rosell, J., Sala, R., Mosquera, M., Made, J.v.d., Sánchez, A., Cuenca, G., Rodríguez, X.P. y Rodríguez, J. 1999. Contribución del yacimiento de Galería (sierra de Atapuerca) al Cuaternario ibérico. In: Atapuerca: ocupaciones humanas y paleoecología del yacimiento de Galería (Eds. E. Carbonell, A. Rosas y J.C. Díez). Arqueología en Castilla y León, memoria 7 , Valladolid, 377-390.

Rothe, P. 1964. Fossile Strausseneier auf Lanzarote. Natur und Museum, 94, 175-218.

Sánchez, A. 1986. Las aves fósiles de La Cuevona (Asturias). Estudios geológicos, 42, 475-478.

Sánchez, A. 1987a. Saalian s.l. avian findings in the northern Iberian Plateau. In: L'évolution des oiseaux d'après le témoignage des fossiles (Coord. C. Mourer-Chauviré). Documents du Laboratoire de Géologie de Lyon, 99, 193-196. 
Sánchez, A. 1987b. Aves fósiles de Atapuerca. In: El hombre fósil de Ibeas y el Pleistoceno de la Sierra de Atapuerca, I (Eds. E. Aguirre, E. Carbonell y J.M. Bermúdez de Castro). Junta de Castilla y León, Valladolid, 67-73+114.

Sánchez, A. 1988. Avifauna. In: La Cueva de Ambrosio (Almería, Spain) y su posición cronoestratigráfica en el Mediterráneo Occidental (Ed. S. Ripoll). British Archaeological Reports, intl.ser., 462, 143-156.

Sánchez, A. 1989. Huéscar-1 (Granada, España): Avance de la lista de aves y consiguientes conjeturas sobre paleoambiente y paleogeografía. In: Geología y paleontología de la cuenca de Guadix-Baza (Eds. M.T. Alberdi y P.F. Bonadonna). Trabajos sobre NeógenoCuaternario, 11, 175-182.

Sánchez, A. 1990a. Aves de los yacimientos mesopleistocenos de Torralba y Ambrona (Soria, España). Acta Salmanticensia, 68, 349-357.

Sánchez, A. 1990b. A new bustard (Otididae, Aves) from the early Pliocene of Layna (Soria, Spain). Paleontologia i Evolució, 23, 223-229.

Sánchez, A. 1995a. Tertiary avian localities of Spain. Acta Universitatis Carolinae Geologica, 39, 719-732.

Sánchez, A. 1995b. Las aves de la unidad estratigráfica TG11 de la Galería (sierra de Atapuerca, Burgos, España). In: Actas de Evolución Humana en Europa y los yacimientos de la Sierra de Atapuerca. Junta de Castilla y León, Valladolid, 137-146.

Sánchez, A. 1996a. The presence of the Waldrapp Geronticus eremita (Plataleidae) in the Plio-Pleistocene boundary in Spain. Ibis, 138, 560-561.

Sánchez, A. 1996b. Aves fósiles del Pleistoceno ibérico: rasgos climáticos, ecológicos y zoogeográficos. Ardeola, 43, 207-219.

Sánchez, A. 1999a. Catálogo paleornitológico del Terciario ibérico y balear. Estudios geológicos, 55, 163-171.

Sánchez, A. 1999b. Nuevas aves fósiles del yacimiento mesopleistocénico de Ambrona (Soria, España). Trabajos de Prehistoria, 56, 115-118.

Sánchez, A. 1999c. Ibis eremita. Restos fósiles hallados en yacimientos españoles. Quercus, 157, 48-49.

Sánchez, A. 1999d. Aves del yacimiento mesopleistoceno de Galería (sierra de Atapuerca). Patrones ecológicos en el Pleistoceno medio. In: Atapuerca: ocupaciones humanas y paleoecología del yacimiento de Galería (Eds. E. Carbonell, A. Rosas y J.C. Díez). Arqueología en Castilla y León, memoria 7, Valladolid, 211-224.

Sánchez, A. 1999e. Implications of the avian fauna for paleoecology in the Early Pleistocene of the Iberian peninsula. Journal of Human Evolution, 37, 375-388.

Sánchez, A. 2000a. Objeciones al caso de los fósiles gibraltareños. Quercus, 170, 52-53.

Sánchez, A. 2000b. Vestigios de una lechuza fósil del Mediterráneo occidental. Quercus, 168, 28-30.

Sánchez, A. 2001. Strigiformes from the Neogene of Spain. Ibis, 143, 146-149.

Sánchez, A., Pérez, B. y Herrero, S. 2000. Passeriformes del Neógeno ibérico: hallazgos en Layna (Plioceno inferior). Revista Española de Paleontología, 15, 101-104.
Sanz, J.L. 1989. El ave cretácica de Cuenca. In: La fauna del pasado en Cuenca (Coord. J.L. Sanz). Instituto Juan de Valdés, Cuenca, 145-166.

Sanz, J.L. and Bonaparte, J.F. 1992. A new order of birds (Class Aves) from the lower Cretaceous of Spain. In: Papers in avian paleontology honoring Pierce Brodkorb (Ed. K.E. Campbell). Natural History Museum of Los Angeles County, sc. ser., 36, 39-49.

Sanz, J.L. and Buscalioni, A.D. 1992. A new bird from the early Cretaceous of Las Hoyas, Spain, and the early radiation of birds. Palaeontology, 35, 829-845.

Sanz, J.L. y López-Martínez, N. 1984. The prolacertid lepidosaurian Cosesaurus aviceps Ellenberger y Villalta, a claimed "protoavian" from the Middle Triassic of Spain. Geobios, 17, 747-753.

Sanz, J.L., Bonaparte, J.F. and Lacasa, A. 1988. Unusual early Cretaceous birds from Spain. Nature, 331, 433435.

Sanz, J.L., Chiappe, L.M. and Buscalioni, A.D. 1995. The osteology of Concornis lacustris (Aves: Enantiornithes) from the Lower Cretaceous of Spain and a reexamination of its phylogenetic relationships. American Museum Novitates, 3133, 23 pp.

Sanz, J.L., Chiappe, L.M., Pérez, B.P., Buscalioni, A.D., Moratalla, J.J., Ortega, F. and Poyato, F.J. 1996. An Early Cretaceous bird from Spain and its implications for the evolution of avian flight. Nature, 382, 442-445.

Sanz, J.L., Chiappe, L.M., Pérez, B.P., Moratalla, J.J., Hernández, F., Buscalioni, A.D., Ortega, F., Poyato, F.J., Rasskin, D. and Martínez, X. 1997. A nestling bird from the Lower Cretaceous of Spain: implications for avian skull and neck evolution. Science, 276, 1543-1546.

Sauer, E.G.F. and Rothe, P. 1972. Ratite eggshells from Lanzarote, Canary Islands. Science, 176, 43-45.

Seguí, B. 1996. Les avifaunes fòssils dels jaciments càrstics del Pliocè, Pleistocè i Holocè de les Gimnèsies. Bolletí de la Societat d'Història Natural de les Balears, 39, 2542.

Seguí, B. and Alcover, J.A. 1999. Comparison of paleoecological patterns in insular bird faunas: a case study from the western Mediterranean and Hawaii. Smithsonian Contributions to Paleobiology, 89, 67-73.

Seguí, B., Mourer-Chauviré, C. and Alcover, J.A. 1997. Upper Pleistocene and Holocene fossil avifauna from Moleta cave (Mallorca, Balearic islands). Bolletí de la Societat d'Història Natural de les Balears, 40, 223-252.

Soler, N. i Garcia, L. 1995. Un probable xiulet paleolític a davant Pau (Serinyà, el Pla de 1'Estany). X Col.loqui Internacional d'Arqueologia de Puigcerdà. Puigcerdà, 195-206.

Treviranus, G.R. 1802-1822. Biologie, oder Philosophie der lebenden Natur. Gotinga, Johann Friedrich Röwer, 6 vols.

Tyrberg, T. 1998. Pleistocene birds of the Palearctic: a catalogue. Publications of the Nuttall Ornithological Club, 27, $720 \mathrm{pp}$.

Tyrberg, T. 1999. Seabirds and Late Pleistocene marine environments in the northeast Atlantic and the Mediterranean. Smithsonian Contributions to Paleobiology, 89, 139-157. 
Tyrberg, T. and Hernández, F. 1995. First fossil record of the Great skua. Bulletin of the British Ornithologists' Club, 115, 167-168.

Uerpmann, H.P. 1971. Die Tierknochenfunde aus der Talayot-Siedlung von S'Illot (San Lorenzo/Mallorca). Studien über frühe Tierknochenfunde von der Iberischen Halbinsel, 2, $111 \mathrm{pp}$.

Uerpmann, H.P. und Uerpmann, M. 1973. Tierknochenfunde aus der phönizischen Faktorei von Toscanos und anderen phönizisch becinflussten Fundorten der Provinz Málaga in Südspanien. Studien über frühe Tierknochenfunde von der Iberischen Halbinsel, 4, 35-100.

Vidal, L.M. 1902. Sobre la presencia del tramo Kimmeridgiense en el Montsech y hallazgo de un batracio en sus hiladas. Real Academia de Ciencias y Artes de Barcelona, 4, 263-274.

Vila, A., Yll, R., Estévez, J., Alcalde, G., Faro, A., Oller, J. i Vilette, Ph. 1985. El Cingle Vermell: assentament de caçadors-recol.lectors del Xè. mil.lenni B.P. Excavacions Arqueològiques a Catalunya, $\mathbf{5}$.

Vilette, P. 1983. Avifaunes du Pléistocène final et de l'Holocène dans le Sud de la France et en Catalogne. Antacina, 11, 190 pp.

Villalta, J. F. de 1963. Las aves fósiles del Mioceno español. Boletín de la Real Sociedad Española de Historia Natural (Geología), 61, 263-285.
Villalta, J.F. de 1964. Datos para un catálogo de las aves fósiles del Cuaternario español. Speleón, 15, 79-102.

Villalta, J. F. de 1965. Un yacimiento villafranquiense en las Islas Medas (prov. de Gerona). Actes IV Congrès International d'Etudes Pyrénéennes, 129-131.

Villalta, J. F. de y Crusafont, M. 1950a. Sobre algunas aves fósiles de Cataluña. Notas y Comunicaciones del Instituto Geológico y Minero de España, 20, 143-156.

Villalta, J.F.de y Crusafont, M. 1950b. Un nuevo yacimiento pleistocénico en Castelldefels. Estudios geológicos, 12, 275-285.

Villaverde, V. y Martínez Valle, R. 1995. Características culturales y económicas del final del Paleolítico Superior del Mediterráneo español. In: Los últimos cazadores (Ed. V. Villaverde). Diputación de Alicante, Alicante, 79-117.

Villaverde, V., Martínez Valle, R., Guillem, P.M., Badal, E., Zalbidea, L. y García, R. 1997. Els nivells Magdalenians de la cova de les Cendres (Teulada, Moraira). Resultats del sondeig del quadre A-17. Aguaits, 13/14, 77-115.

Vuilleumier, F. 1975. Zoogeography. Avian Biology, 5, 421496.

Walker, C.A., Wragg, G.M. and Harrison, C.J.O. 1990. A new shearwater from the Pleistocene of the Canary islands and its bearing on the evolution of certain Puffinus shearwaters. Historical Biology, 3, 203-224.

Manuscrito recibido: 22 de mayo, 2000 Manuscrito aceptado: 24 de octubre, 2000 